Check for updates

Cite this as: $B M J 2021 ; 372: n 236$ http://dx.doi.org/10.1136/bmi.n236 Published: 03 February 2021

\section{Update to living systematic review on prediction models for diagnosis and prognosis of covid-19}

This living systematic review by Wynants and colleagues (BMJ 2020;369:m1328) has been updated. For the latest update, visit doi:. The latest version of this living systematic review critically appraises 232 prediction models for diagnosis and prognosis of coronavirus disease 2019 (covid-19), of which 87 were added in the latest update. Summary statistics on study characteristics, model availability, and model quality are also presented. All extracted data per model are publicly available at https://www.covprecise.org/living-review/. The quality of published prediction models is gradually improving but all models remain at high or unclear risk of bias, suggesting that their reported performance is likely optimistic and might not be met if these models are applied in daily medical practice. This update shows two promising prediction models (the Jehi et al diagnostic model and the prognostic $4 \mathrm{C}$ Mortality Score), both derived from large databases, which should be validated by independent researchers. 